\title{
Die Oberflächenveredelungs- industrie in Corona-Zeiten
}

\author{
Als Teil der global agierenden Industrie und auch Querschnittsindustrie über zahlreiche \\ Anwendungsgebiete hinweg äußert die Industrie der Oberflächenveredelung große Besorgnis. Dies \\ ergab eine Kurzumfrage des Verbands für die Oberflächenveredelung von Aluminium e. V. (VOA).
}

\begin{abstract}
Alle Industrielenker weltweit sind gefordert, ihre Unternehmen und ihre Mitarbeiter durch den dynamisch ablaufenden, kaum vorhersehbaren und möglicherweise gesundheitsschädigenden Prozess der Coronapandemie zu führen und zum Wohle ihres Unternehmens zu handeln. Nach dem zweiten Weltkrieg hat es keinen Einbruch gegeben, der mit dem derzeit weltweit vergleichbar ist. Nach Auffassung des VOA geht es darum, alle Anstrengungen zu unternehmen, die Arbeitsplätze zu erhalten und die wirtschaftliche Betätigung in der Zukunft weiter zu führen; gleichzeitig aber auch auf die Unversehrtheit der Gesundheit zu achten.

Die Kurzumfrage des VOA wurde sowohl unter den Ordentlichen Mitgliedern $(\mathrm{OM})$, die eloxieren, beschichten und entlacken, als auch unter den Fördermitglie-
\end{abstract}

dern (FÖM), beispielsweise aus der Chemie oder Pulverherstellung, durchgeführt. $90 \%$ der OM und $100 \%$ der FÖM zeigten sich besorgt über die Folgen der Coronapandemie. Die Mitglieder äußerten, dass ein Bestell- und Umsatzrückgang infolge der geminderten Kapazitätsauslastung aus den Bereichen Automotive, Maschinenbau, Architektur und Flugzeugbau zu verzeichnen ist $(43,8$ und 56,3\% OM, jeweils $75 \%$ FÖM). Auch machen den Mitgliedsunternehmen unterbrochene Lieferketten $\mathrm{zu}$ schaffen (18,8 \% OM, 12,5 \% FÖM). Daraus folgt, dass seitens der Unternehmen der Produktionsprozess angepasst und bei den OM in 46,9 \% der Fälle Kurzarbeit angemeldet wurde, in 28,1\% der Unternehmen ist die Kurzarbeit bereits eingeführt. Bei den FÖM sind es immerhin 37,5 \%. Entlassungen gab es nur bei 6,3\% der teilnehmenden
Unternehmen (OM). Wieder einmal zeigt sich, dass sich das typisch deutsche Instrument der Kurzarbeit bewährt und den Unternehmen ermöglicht, den eingearbeiteten Mitarbeiterstamm zu halten.

Alle Unternehmen haben die Hygienevorschriften (OM 96,9 \% , FÖM 100 \%) erweitert, schulen die Mitarbeiter zu den Hygienestandards (OM 62,5\%) und überprüfen die Einhaltung der gesetzten Standards (OM und FÖM 62,5 \%). Die Maßnahmen gehen bis zur Bereitstellung von Schutzkleidung, Anpassung der Schichtmodelle, Schließung von Betriebskantinen, Ausweitung des Home Office, Reiseverbote für Mitarbeiter und Ausschluss fremder Personen aus den Unternehmen. Die Maßnahmen haben dazu beigetragen, die akute Infektion durch das Coronavirus einzudämmen. VOA-Geschäftsführerin Dr. Alexa A. Becker

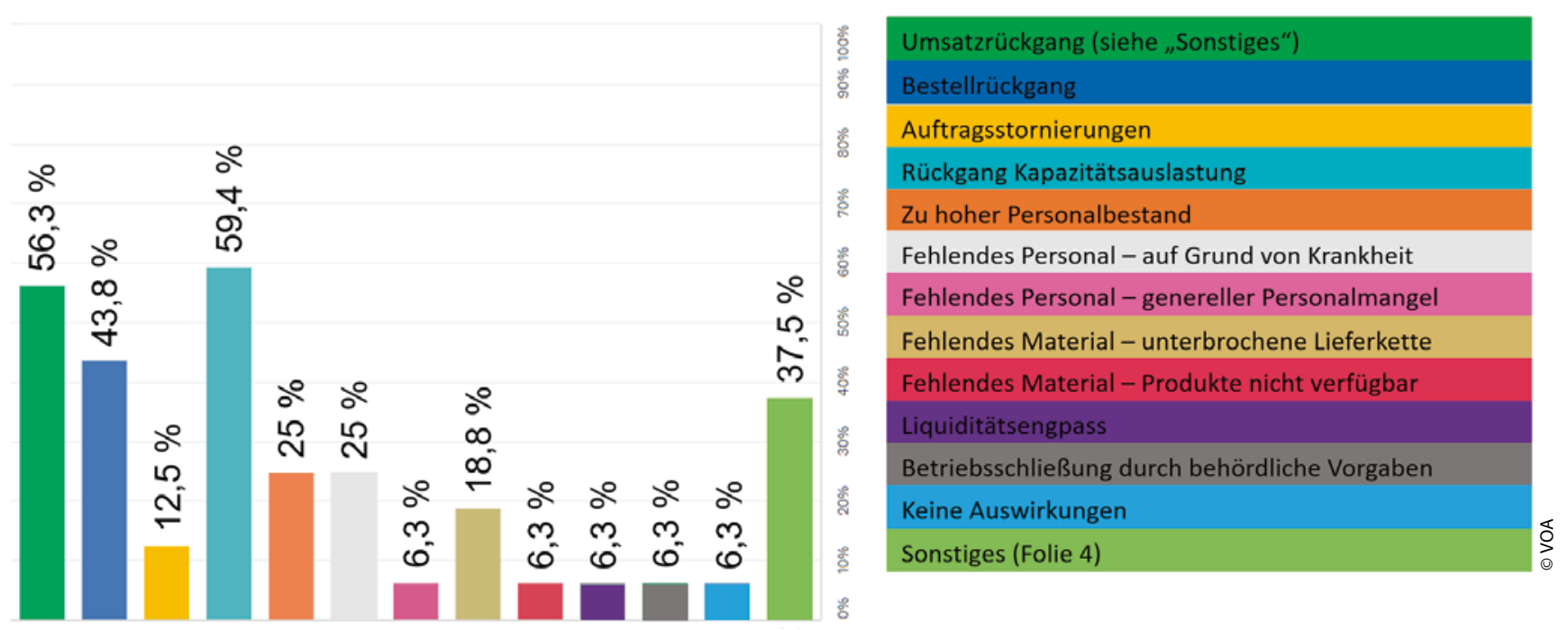

Welche Auswirkungen hat die Corona-Pandemie auf den Geschäftsbetrieb? Ergebnisse der Befragung von Ordentlichen Mitgliedern des VOA. 

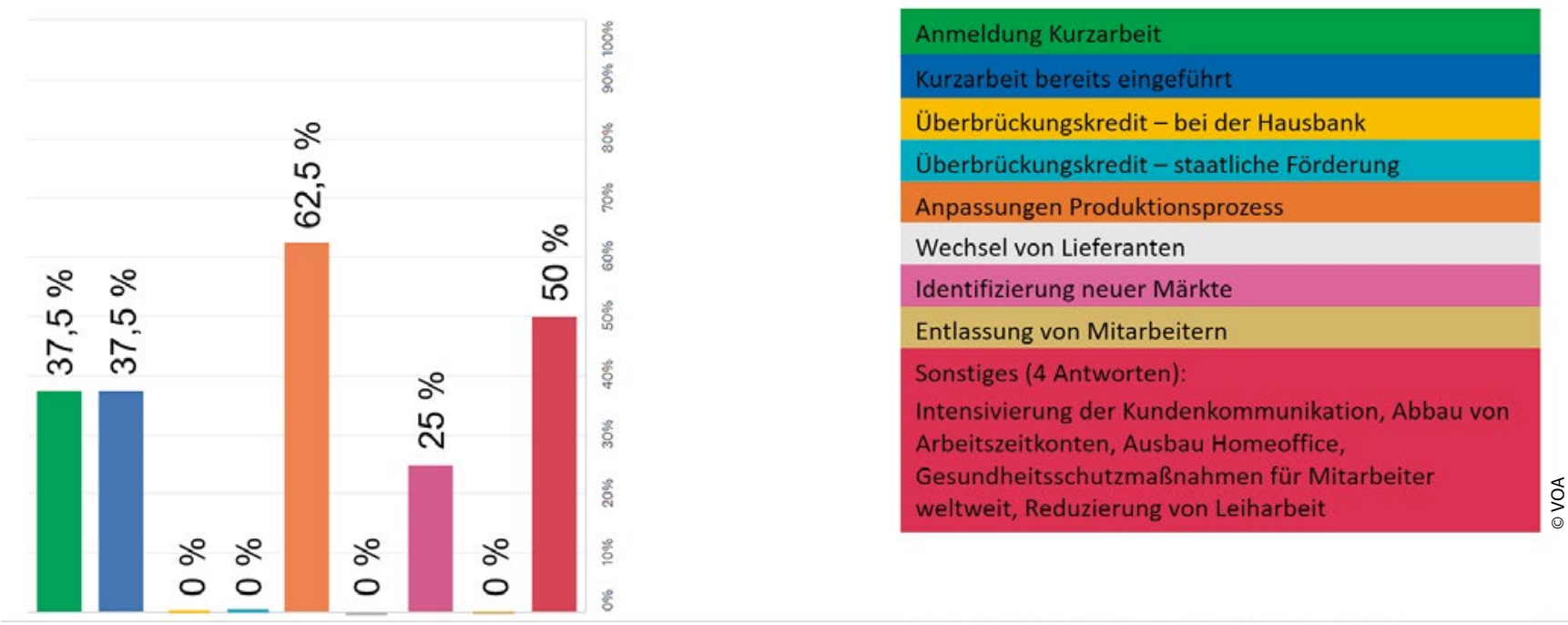

Welche Maßnahmen zur Dämpfung der wirtschaftlichen Schäden wurden ergriffen? Ergebnisse der Befragung von Fördermitgliedern des VOA.
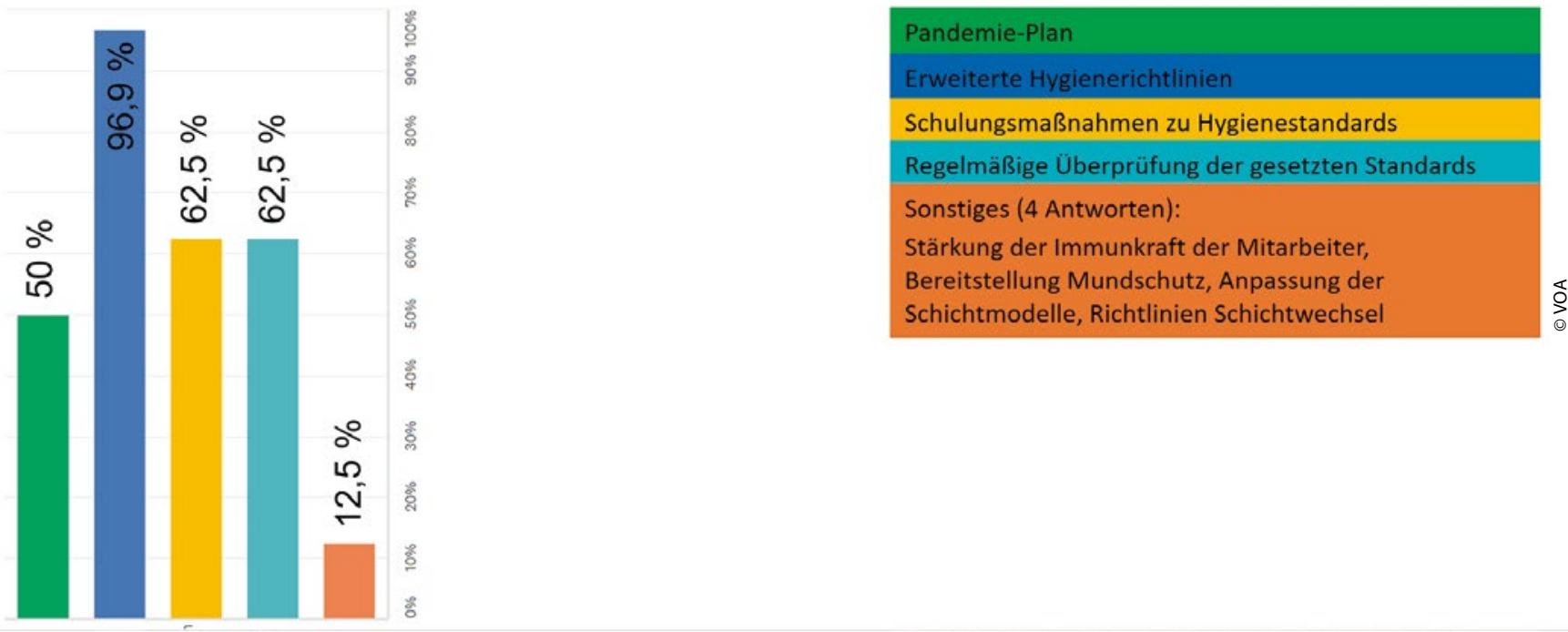

Welche sonstigen Maßnahmen wurden im Unternehmen auf Grund der Corona-Pandemie ergriffen? Ergebnisse der Befragung von Ordentlichen Mitgliedern des VOA.

stellt fest: „Jedem ist bewusst, dass eine Normalität erst dann wieder einkehren kann, wenn alle Mitbürger weltweit mit einem Impfstoff oder Medikamenten gegen das Coronavirus versorgt werden können.“ Ein Konzept für einen politisch und gesellschaftlich akzeptierten Fahrplan zum globalen Lockout der Wirtschaft bedingt die Verantwortung jedes einzelnen. Nur dann, wenn Gesundheitsschutz und Wirtschaftsinteressen in Einklang gebracht werden können, gelingt es auch den Unternehmen der Oberflächenveredelungsindustrie wie der gesamten Wirtschaft wieder, die Produktion voll anzufahren und damit die Arbeitsplätze und die Existenz sichern.

Becker: „Die Politik tut viel, diese Ziele zu erreichen und die Oberflächenveredelungs- industrie weiß die Bemühungen zu würdigen. Nun gehört zu den kurzfristigen Maßnahmen, dass beispielsweise Rückzahlungsfristen im Falle der Stundung von Sozialversicherungsbeiträgen verlängert werden." Auch die Beeinträchtigung grenzüberschreitender Lieferketten und Einschränkungen beim Pendlerverkehr seien sinnvoll zu stoppen; zudem müsste es Erleichterungen beim Zoll und eine unbürokratische Außenwirtschaftspolitik geben. Darüber hinaus sollte in dieser außergewöhnlichen Zeit ein Strukturpaket verabschiedet werden, um die Wirtschaft zu stärken. Gerade in der Steuerpolitik ist daran zu denken, dass es keine Steuererhöhungen geben, vielmehr die Körperschaftssteuer gesenkt werden soll- te, Investitionen (Abschreibungen) sollten gefördert und der Soli direkt abgeschafft werden, um den Konsum zu fördern. Alles in allem geht es darum, aus der weltweiten Krise, die die Wirtschaft unvermittelt getroffen hat, so bald wie möglich und so sicher wie nötig heraus zu kommen. //

\section{Kontakt}

\section{VOA}

Verband für die Oberflächenveredelung von Aluminium e.V.

München

www.voa.de 